\title{
ERRATUM
}

\section{INDUCED ELECTROSTATIC SELF-INTERACTION IN THE SPACE-TIME OF A GLOBAL MONOPOLE WITH INNER STRUCTURE}

\author{
[Int. J. Mod. Phys. D 18 (2009) 1085] \\ D. BARBOSA*,‡ , J. SPINELLY ${ }^{\dagger, \S}$ and E. R. BEZERRA DE MELLO*, \\ *Departamento de Física-CCT, \\ Universidade Estadual da Paraiba, \\ Juvêncio Arruda $S / N$, C. Grande, PB, Brazil \\ ${ }^{\dagger}$ Departamento de Física-CCEN, \\ Universidade Federal da Paraíba, 58.059-970, \\ C. Postal 5.008, J. Pessoa, PB, Brazil \\ ¥denis.fisico@fisica.ufpb.br \\ §jspinelly@uepb.edu.br \\ ๑emello@fisica.ufpb.br
}

Received 21 September 2010

In this paper we correct previous result obtained in published paper.

In the analysis of electrostatic self-energy on a point-like electric-charged particle induced by a global monopole space-time considering the ballpoint pen model for the region inside, ${ }^{1}$ we found, among several other interesting results, that near the boundary this self-energy presents logarithmic divergent results in both regions, i.e. for $r \leq r_{0}$ and $r \geq r_{0}$, where $r_{0}$ is the radius of monopole. However, in recent paper Ref. 2, we have observed that these conclusions are not correct. In fact, in the analysis for large value of $l$, of the coefficients inside the summations of Eqs. (44) and (56) of Ref. 1, we have missed out extra terms in both expressions. These extra terms exactly cancel the $1 / l$ behavior previously obtained, giving as correct expressions, coefficients proportional to $1 / l^{2}$, which provide finite results for $r=r_{0}$. So, we conclude that there is no logarithmic divergent contribution on the induced self-energy in the ballpoint pen model.

The rest of our results, especially the most important one related with the behavior of self-energy near the monopole's center, and also the behavior of selfenergy for large distances from the monopole, remain correct. 


\section{Acknowledgment}

We would like to thank Aram A. Saharian for helpful discussion.

\section{References}

1. D. Barbosa, J. Spinelly and E. R. Bezerra de Mello, Int. J. Mod. Phys. D 18 (2009) 1085 .

2. D. Barbosa, U. de Freitas and E. R. Bezerra de Mello, Induced self-energy on a static scalar charged particle in the spacetime of a global monopole with finite core, arXiv:1009.0195 [hep-th]. 